\title{
Contrast-Enhanced Ultrasound for the Assessment of Pancreatic Lesions
}

\author{
Roxana Șirli and Alina Popescu \\ Additional information is available at the end of the chapter
}

http://dx.doi.org/10.5772/65066

\begin{abstract}
Transabdominal ultrasound (US) is the first-line imaging method used to diagnose pancreatic lesions, but contrast techniques are needed to differentiate among inflammatory and malignant lesions, as well as between pseudocysts and cystic tumors. Contrast-enhanced (CE) ultrasonography has been proven to be a useful tool in this regard with performance similar to contrast-enhanced computer tomography/magnetic resonance imaging (CT/MRI), being also safer and nonirradiant. According to the EFSUMB guidelines on the nonhepatic use of contrast-enhanced ultrasound (CEUS), this method is useful to improve characterization of ductal adenocarcinoma; to differentiate between pseudocysts and cystic tumors; to differentiate vascular (solid) from avascular (liquid/necrotic) components of a lesion; to better define the dimensions and margins of a lesion, including its relationship with adjacent vessels; and to help the choice for a next imaging technique.
\end{abstract}

Keywords: contrast-enhanced ultrasound, pancreas, pancreatic adenocarcinoma, pancreatic cystic lesions

\section{Introduction}

The pancreas, a retroperitoneal organ, is more difficult to evaluate by ultrasound (US), mostly due to a poor acoustic window generated by the interposition of intestinal gas between the transducer and the pancreas. In order to be able to correctly evaluate the pancreas, an experienced operator is needed. 
On the other hand, US is a useful tool to identify pancreatic lesions, but ultrasound alone is not enough for the differential diagnosis and staging of the identified lesions, especially if a malignant tumor is suspected.

Contrast-enhanced ultrasound (CEUS) is not useful for the detection of focal pancreatic lesions, either solid or liquid. It is useful for the characterization of ultrasound-detected lesions at this level [1]. The technique can be used in acute and chronic pancreatitis (CP), in the characterization of solid tumors: ductal adenocarcinoma or neuroendocrine tumors, in the characterization of pseudocysts or pancreatic cystic tumors.

When a pancreatic tumor is detected, an immediate and correct differential diagnosis is mandatory to establish the appropriate management [2]. Conventional ultrasound followed by CEUS can provide a rapid assessment of the pancreatic lesion's pattern and can characterize the vascularization, thus making possible a differential diagnosis immediately after detection.

CEUS has the advantage of being a real-time imaging method that allows continuous visualization of vascular enhancement pattern, as opposed to contrast-enhanced (CE) computer tomography (CT) or magnetic resonance imaging (MRI), which only take snapshots at preset time moments. Due to lack of side effects and irradiation, CEUS can be repeated immediately if inconclusive.

The most frequently used ultrasound contrast agent for pancreas examination is SonoVue (Bracco SpA, Milan, Italy), a second generation, strictly intravascular contrast agent, containing sulfur hexafluoride-filled microbubbles encapsulated in a phospholipid shell. US contrast agents have no influence on micro-vascularity and can be also used in patients with renal failure, and also allergic reactions are exceptionally rare [2,3], as opposed to contrast-enhanced CT/MRI [4-7].

\section{CEUS technique and CEUS aspect of the normal pancreas}

For the CEUS examination of the pancreas, usually a $2.4 \mathrm{ml}$ bolus of SonoVue is injected in an antecubital vein, followed by a $10 \mathrm{ml}$ bolus saline solution. Contrast-specific US modes are required for the contrast study. They are available on specific ultrasound systems and are generally based on the cancellation and separation of linear US signals from the tissue and use of the nonlinear response from the contrast agent microbubbles [1].

The examination is performed with low mechanical index, using conventional image for orientation, and following in the same time the contrast study in a specific window. A "realtime" dynamic observation of the contrast-enhanced phases - arterial (early stage of enhancement, until $30 \mathrm{~s}$ ) and late (delayed stage of enhancement, 30-45 until $120 \mathrm{~s}$ following contrast injection) - begins immediately after the contrast bolus [1]. The following aspects are followed up during CEUS: timing and intensity of enhancement and the distribution of the contrast agent. 
Approximately 25-40 s following the contrast bolus, the pancreas shows a homogeneous intense enhancement due to its rich vascularity (Figure 1) [8]. Also due to the rich vascularity, the pancreas has a rapid "wash-out," and 2 min following the contrast bolus, the pancreas appears as hypoenhancing as compared to the nearby liver (Figure 2) [8]. Thus, CEUS is useful especially for delineation of avascular pancreatic lesions.

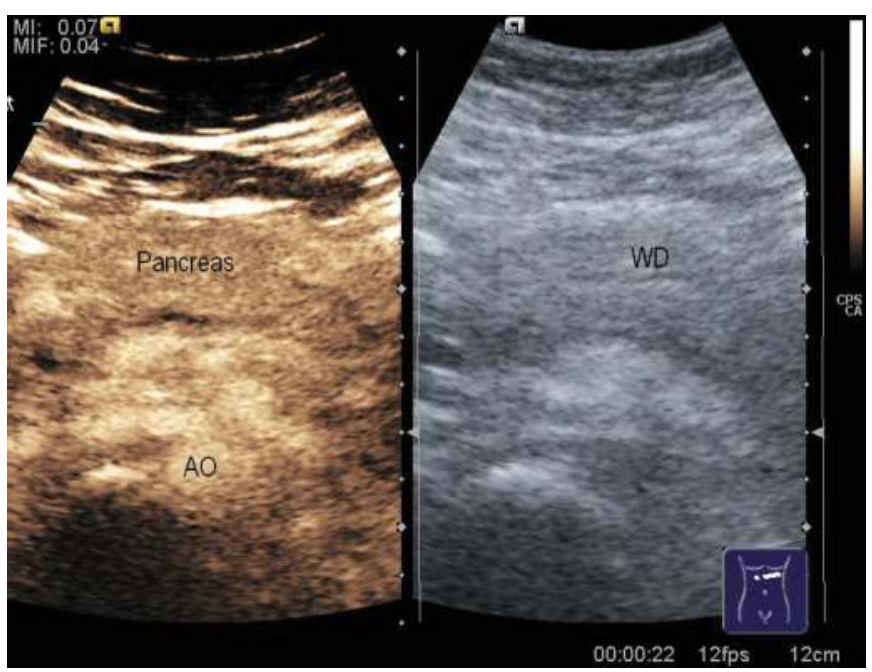

Figure 1. CEUS of the normal pancreas, arterial phase: homogeneous intense enhancement (AO-aorta).

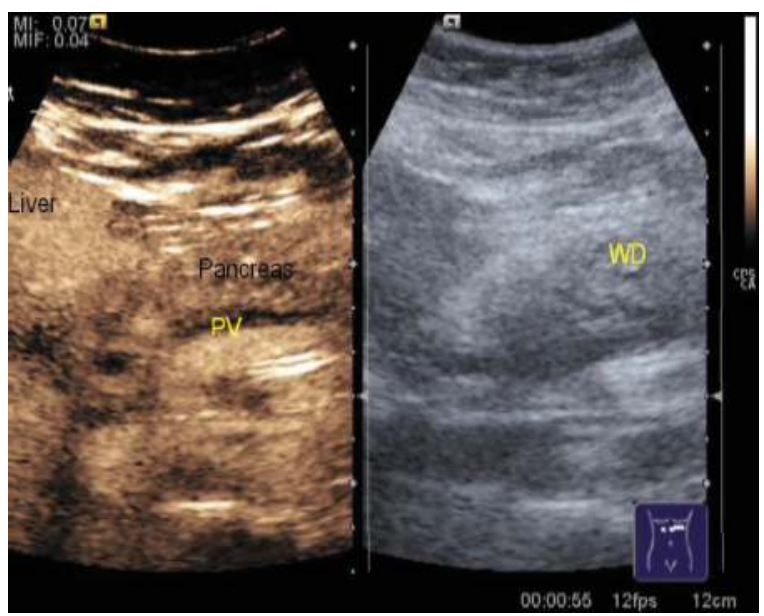

Figure 2. CEUS of the normal pancreas, late phase: the pancreas is hypoenhancing as compared to the liver (PV-portal vein; WD-Wirsung duct). 


\section{Acute pancreatitis (AP)}

$\mathrm{AP}$ is a potentially severe disease with unpredictable outcome which can develop multiple complications with fast dynamics. The early differential diagnosis between a mild, edematous form and a severe, necrotic-hemorrhagic one is very important in order to be able to adapt the treatment and to be able to try to prevent the occurrence of complications. Currently, contrastenhanced CT (CE-CT) is considered to be the reference method for the assessment and for staging AP [9]. But CE-CT is an irradiating, relatively expensive technique, and animal studies have suggested a potential risk of aggravation of AP following CE-CT due to pancreatic microcirculation impairment by the contrast agent [10-12], even if in human studies this effect has not been proven $[13,14]$. Another impediment for CE-CT is the need for repetitive examinations according to the patient's evolution. This is why a safer, cheaper diagnostic tool would be useful for the diagnosis, staging, and follow-up of patients with AP.

Abdominal US is in most cases the first imaging method used to evaluate patients with AP since it is widely available, safe, rapid, and inexpensive. It is also nonirradiant, and thus, it can be repeated as often as needed to follow-up the patient's evolution. On the other hand, US has limitations due to the poor acoustic window in AP secondary to large amount of bowel gas and also due to the patient's abdominal pain which makes him unable to cooperate for an optimal evaluation.

Standard abdominal US allows only assessment of the imaging aspect of the pancreas in AP, without being able to assess vascularity. But it also reveals suggestive signs for a severe AP such as hyperechoic bursa omentalis (Figure 3) and presence of intra-peritoneal collections (peripancreatic, pericolic or in the Douglas space), while using Doppler US, a splenic vein thrombosis may be seen.

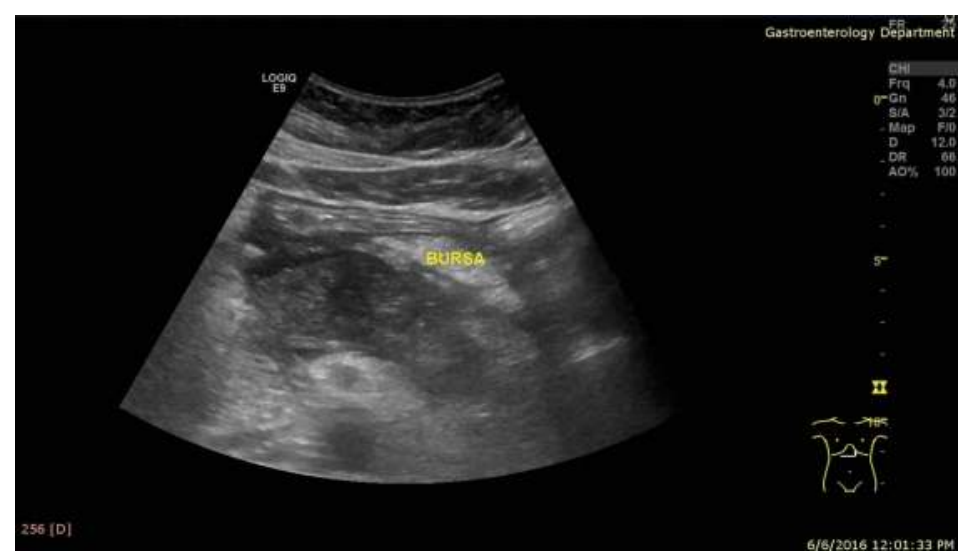

Figure 3. Acute pancreatitis, standard US: hypoechoic, inhomogeneous enlarged pancreas, hyperechoic bursa omentalis. 
CEUS allows visualization of pancreatic vascularity and thus is able to reveal necrotic areas which will not enhance following the contrast bolus (Figure 4). But the same limitations as for standard US apply for CEUS, which is useless if the acoustic window for the pancreas is poor.

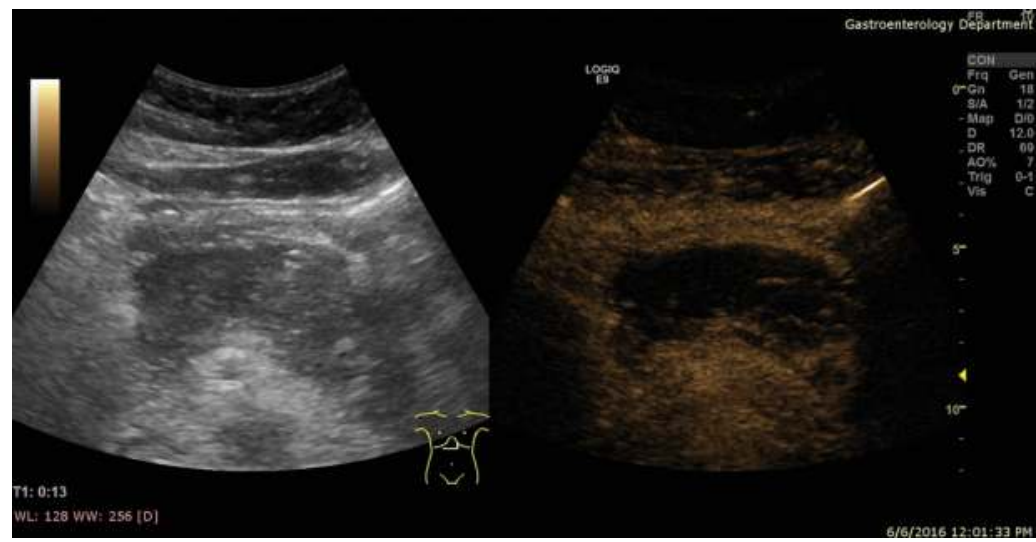

Figure 4. Severe acute pancreatitis, CEUS, arterial phase: almost the entire pancreas is unenhancing, revealing extensive necrosis.

Rickes et al. evaluated the accuracy of CEUS for the diagnosis of AP severity, considering CE$\mathrm{CT}$ as the reference method, and they found out that CEUS had $82 \%$ sensitivity (Se), $89 \%$ specificity (Sp), 95\% positive predictive value (PPV), and 67\% negative predictive value (NPV) for diagnosing severe AP, with a much lesser cost than CE-CT [15]. Similar results were obtained by other authors, CEUS Se and Sp ranging from 86 and 97\%, respectively [16], to 90.3 and $98.8 \%$, respectively (97.4\% accuracy) [17].

Thus, all these studies confirmed the value of CEUS for detecting pancreatic necrosis and for predicting the severity of AP. It showed similar results as compared with CE-CT with fewer side effects since CEUS is nonirradiant and since the US contrast agents have no influence on microvascularity and can also be used in patients with renal failure.

\section{Chronic pancreatitis (CP)}

During the evolution of $\mathrm{CP}$, inflammatory masses can appear, a characteristic feature of pseudotumoral CP. Differential diagnosis between this entity and pancreatic cancer is often difficult not only due to the similar imaging aspect but also due to similar clinical symptoms [18]. The US aspect of pseudotumoral CP is most often of a hypoechoic, imprecisely delineated mass in the head of the pancreas. CEUS is useful to differentiate among the two entities since pancreatic adenocarcinomas are hypoenhancing following contrast (due to massive desmoplastic reaction as well as poor vascularity), while the inflammatory pseudotumor in CP will be enhancing in the arterial phase [19-21]. 
D'Onofrio et al. evaluated the performance of CEUS to diagnose pseudotumoral CP in a study that included 173 pancreatic masses. CEUS had 88.6\% Se, 97.8\% Sp, 91.2\% PPV, and 96\% accuracy for the diagnosis of pseudotumoral CP, while in $94 \%$ of cases the inflammatory mass showed moderate enhancement following contrast, similar to the adjacent pancreatic parenchyma [20].

A more recent study showed that the blood flow ratio between the superior mesenteric artery and the pancreatic parenchyma evaluated by CEUS correlates with the grade of chronic pancreatitis and concluded that this safe and convenient method may be useful for the early diagnose of $\mathrm{CP}[22]$.

A special entity is autoimmune pancreatitis. It is characterized by a high level of gammaglobulins or IgG, presence of auto-antibodies, mild or absent clinical symptoms. Imaging techniques reveal an enlarged, "sausage-like" pancreas, with diffuse, irregular thinning of the Wirsung duct (WD), with possible stenosis of the retro-pancreatic main biliary duct and rarely cysts or calcifications in the pancreatic parenchyma [20]. Autoimmune pancreatitis is sometimes associated with other autoimmune diseases such as diabetes mellitus, inflammatory bowel disease, primary biliary cirrhosis, primary sclerosing cholangitis, or lupus.

On standard US, in autoimmune pancreatitis the imaging changes are most often diffuse. The pancreas is enlarged, hypoechoic and with a compressed Wirsung duct. Following contrast bolus, the pancreas is moderate or hyperenhancing in the early arterial phase, but also with inhomogeneous enhancement due to lymphocytic infiltration and fibrosis [20].

\section{Solid focal pancreatic lesions}

\subsection{Ductal adenocarcinoma}

Ductal adenocarcinomas represent $80-90 \%$ of the exocrine pancreatic tumors [23]. They have a poor prognosis, due to both their aggressiveness and the difficult diagnosis in early phases, in which effective treatment can be initiated.

On standard US, ductal adenocarcinoma most frequently appears as a hypoechoic, imprecisely delineated mass which sometimes exceeds the contour of the pancreas (which facilitates detection) (Figure 5), but which other times is completely embedded in the pancreatic parenchyma.

In CEUS, ductal adenocarcinoma is only slightly enhancing in the early phase, appearing as hypoenhancing as compared with the adjacent pancreatic parenchyma (Figure 6) probably due to the desmoplastic reaction and low mean vascular density [20, 23-25]. This pattern is present in approximately $90 \%$ of cases $[2,20,26]$. Moreover, CEUS enables a better visualization and staging of ductal adenocarcinoma by allowing a better delineation as well as assessment of vascular invasion [23, 27-29]. 


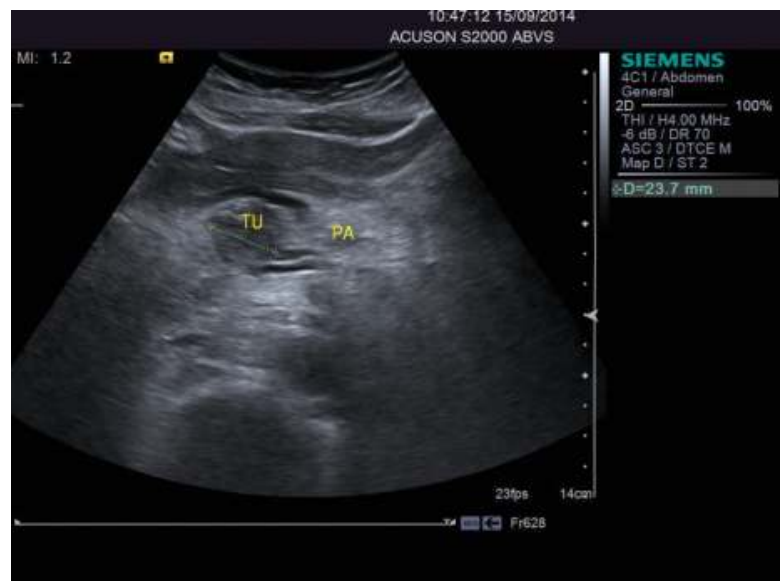

Figure 5. Ductal adenocarcinoma in the head of the pancreas, standard US: hypoechoic mass (TU arrows). PApancreas.

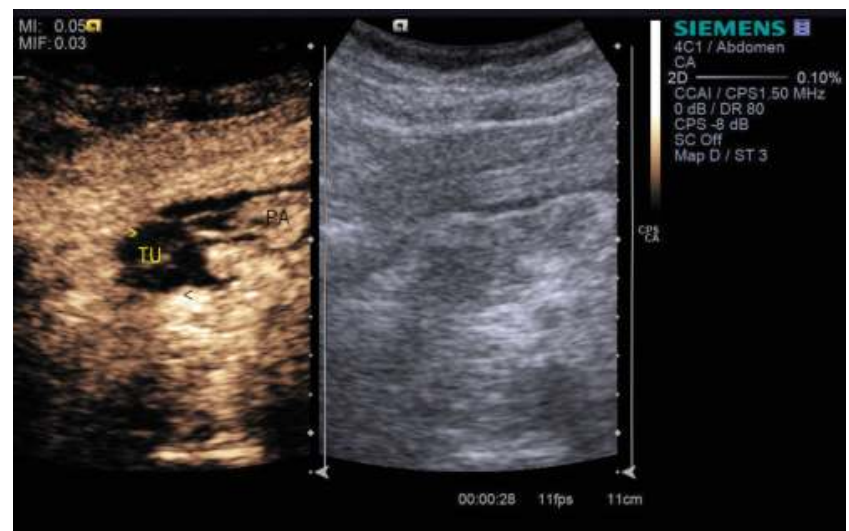

Figure 6. Ductal adenocarcinoma in the head of the pancreas, CEUS-arterial phase: hypoenhancing mass (TU-arrows) as compared to the adjacent pancreatic parenchyma (PA).

Also CEUS enables liver assessment in the late vascular phases, thus allowing visualization of eventual metastases, with a better accuracy than standard US. In the late phase (more than $120 \mathrm{~s}$ after the contrast bolus), liver metastases will appear as hypoenhancing focal liver lesions.

\subsection{Neuroendocrine tumors}

Neuroendocrine tumors may be symptomatic if they are secreting, with specific clinical signs according to the secreted hormone, or asymptomatic if they are nonfunctioning, in this case only nonspecific symptoms are present, secondary to tumoral growth. 
Neuroendocrine tumors are hypervascular lesions [30]. On standard US, they have a similar aspect to ductal adenocarcinoma, as hypoechoic masses (Figure 7). The differential diagnosis among the two entities is extremely important, for prognosis, as well as for therapeutic strategy, and this is where CEUS can make a difference.

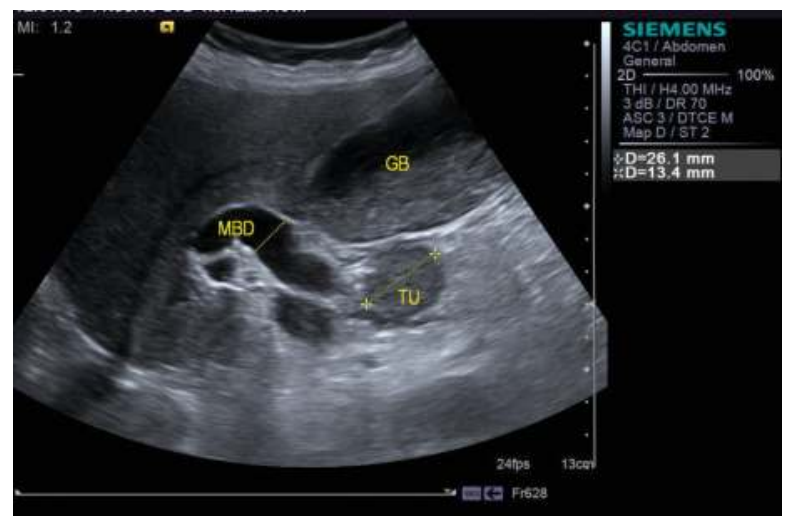

Figure 7. Neuroendocrine tumor in the pancreatic head, standard US: hypoechoic mass (TU). Dilated main biliary duct (MBD). GB - gallbladder.

On CEUS, there are different enhancing patterns according to the size and tumor vascularity [23]. Thus, large tumors show an intense enhancement in the early phase, excepting the necrotic areas that are unenhancing, while medium-sized lesions will also by enhancing in the arterial phase (Figure 8) [31]. Both types of lesions are hypoenhancing in the late phase (Figure 9). On the other hand, nonfunctioning neuroendocrine tumors can be hypovascular due to their dense hyaline stroma (they also appear as hypointense on CE-CT) [31, 32].

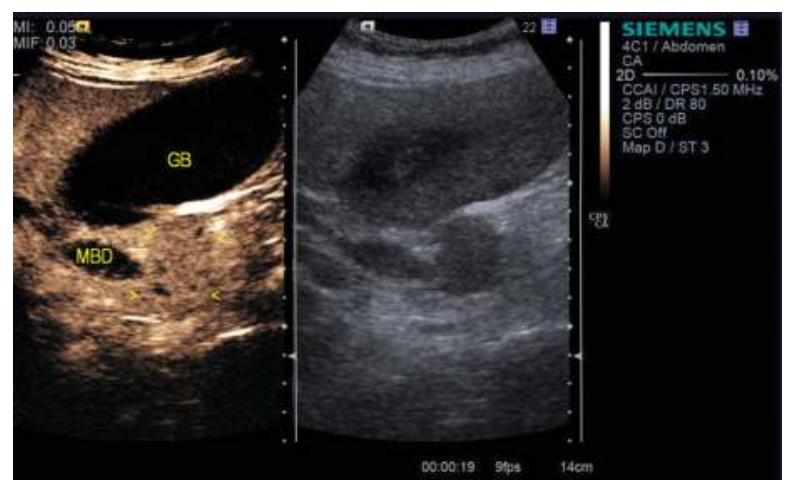

Figure 8. Neuroendocrine tumor in the pancreatic head, CEUS-arterial phase: hyperenhancing mass (arrows). Dilated main biliary duct (MBD). GB-gallbladder. 


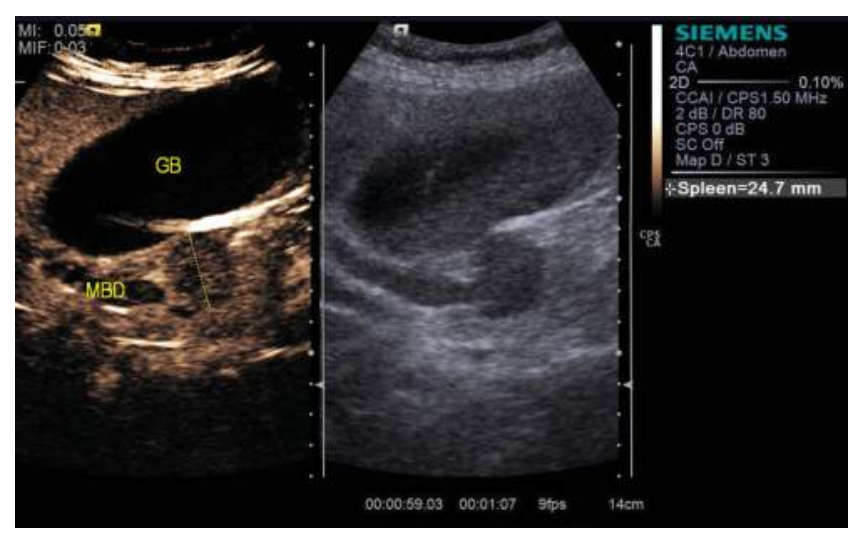

Figure 9. Neuroendocrine tumor in the pancreatic head, CEUS-late phase: hypoenhancing mass (arrows). Dilated main biliary duct (MBD). GB - gallbladder.

\section{Cystic focal pancreatic lesions}

\subsection{Pseudocysts}

Pseudocysts are the most frequent cystic pancreatic lesions and are characterized by a fibrous wall with no epithelium [33]. On standard US, they usually appear as anechoic lesions in the pancreatic area. Sometimes echoic material can be seen inside the lesions, usually in the lower areas (Figure 10). Association with an episode of acute pancreatitis in the recent history of the patient can be useful for a positive diagnosis.

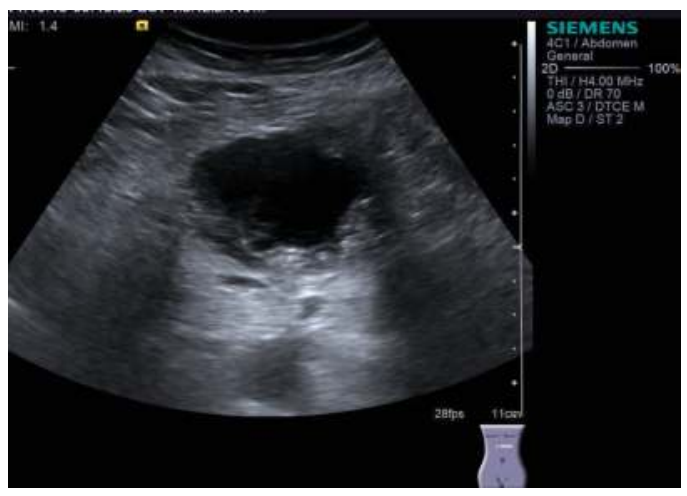

Figure 10. Pancreatic cystic lesion, standard US. Anechoic well-defined lesion, with thick, irregular walls that also have echoic protrusions. 
In CEUS, pseudocysts appear as completely unenhancing due to the fact that they are avascular structures, even if in standard US they may have an echoic content (Figure 11). This feature is very important for the differential diagnosis with cystic pancreatic tumors [2, 23, 34]. The method has up to $100 \%$ sensitivity and specificity in characterizing these lesions [34].

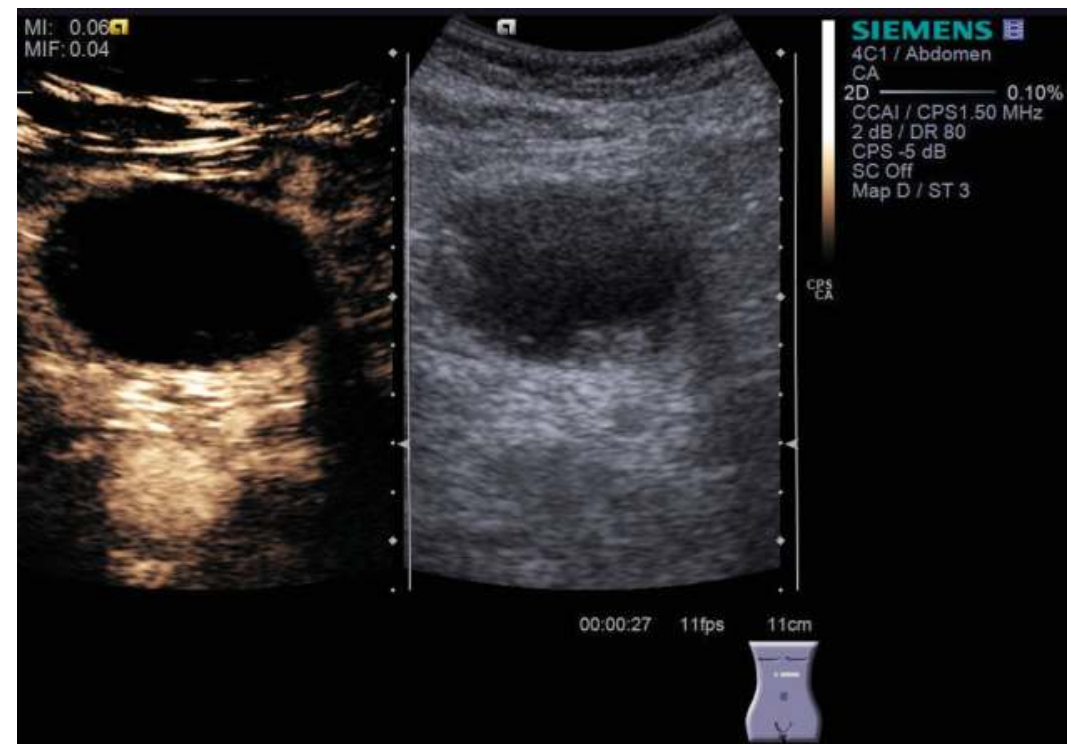

Figure 11. Pancreatic cystic lesion the same as in Figure 10, CEUS-arterial phase. Anechoic content with unenhancing walls and protrusions. Definitive diagnosis: pancreatic pseudocyst.

\subsection{Mucinous cystadenomas and cystadenocarcinomas}

Mucinous cystadenomas of the pancreas are rather rare and considered to be premalignant lesions. If found they should be resected to avoid malignant transformation. Differentiation between pancreatic serous cystadenoma and mucinous cystadenoma is difficult and is based on fine needle aspiration (FNA) that will reveal in the later high levels of carcinoembryonic antigen (CEA) in the aspirated fluid as well as a low level of pancreatic enzymes $[35,36]$. They are cystic pancreatic masses, usually multilocular, rarely unilocular when they must be differentiated from pancreatic pseudocysts and from serous cystadenomas [37-41].

On standard US, mucinous cystic tumors appear as cystic masses with thick wall and septa, with echoic protrusions from the wall and with the content (mucin) not always anechoic, sometimes with peripheric calcifications (Figure 12). On CEUS, the cystic wall as well as the septa will enhance following the contrast bolus, being easy to differentiate from the unenhancing content (Figure 13). The presence of enhancing walls, protrusions, and septa is the differential element from pancreatic pseudocysts [23, 41-43]. 


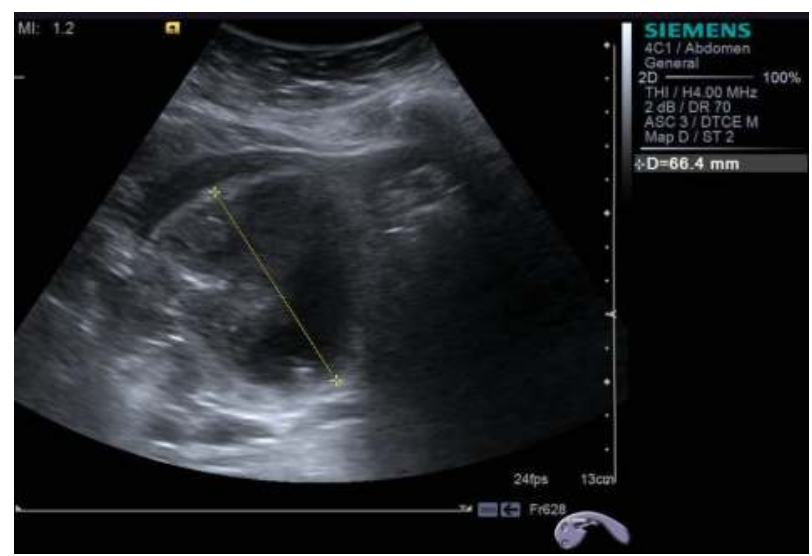

Figure 12. Large lesion in the body of the pancreas, standard US: lesion with thick walls and mixed content: anechoic component as well as echoic protrusion.

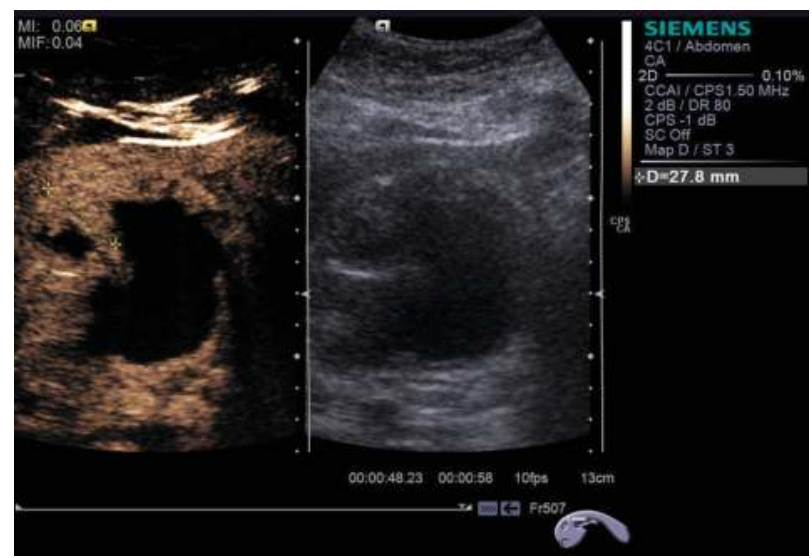

Figure 13. Large lesion in the pancreatic body: CEUS - arterial phase: the walls and the protrusion are enhancing. Conclusive for cystic tumor of the pancreatic body.

The diagnosis of intra-ductal papillary mucinous neoplasms (IPMN), main-duct or side branch-duct types, is based on MRI and endoscopic ultrasound. CEUS can be also helpful for differentiating between perfused (tumoral) and nonperfused (clot) regions [44].

\subsection{Serous cystadenomas}

Serous cystadenomas are benign cystic lesions, with a lobulated microcystic structure with thin and centrally oriented septa, which are vascularized on CEUS [43]. It may mimic a solid lesion, both on conventional US and on CEUS, but they are hyperenhanced on CEUS [44]. 
The criteria used for the differential diagnosis of pancreatic masses (standard US, Doppler US and CEUS) are presented in Table 1.

\begin{tabular}{|c|c|c|c|}
\hline Lesion & Standard US & Power Doppler US & CEUS \\
\hline $\begin{array}{l}\text { Ductal } \\
\text { adenocarcinoma }\end{array}$ & $\begin{array}{l}\text { Hypoechoic } \\
\text { Imprecisely delineated } \\
\text { Dilated Wirsung duct } \\
\text { Vascular invasion } \\
\text { Metastases }\end{array}$ & $\begin{array}{l}\text { Undetectable } \\
\text { tumoral } \\
\text { vessels }\end{array}$ & $\begin{array}{l}\text { Poorly vascularized tumor } \\
\text { (hypoenhancing) } \\
\text { Marginal tumoral vessels }\end{array}$ \\
\hline Acute pancreatitis & $\begin{array}{l}\text { Hypoechoic } \\
\text { Imprecisely delineated } \\
\text { Necrotic areas } \\
\text { Thrombosis }\end{array}$ & $\begin{array}{l}\text { Rarely detectable } \\
\text { vessels }\end{array}$ & $\begin{array}{l}\text { Enhancement according to } \\
\text { necrosis and inflammation: } \\
\text { hyperenhancement in edematous } \\
\text { AP with unenhancement of the } \\
\text { necrotic areas }\end{array}$ \\
\hline Chronic pancreatitis & $\begin{array}{l}\text { Dilated Wirsung duct } \\
\text { Calcifications }\end{array}$ & $\begin{array}{l}\text { Rarely detectable } \\
\text { vessels }\end{array}$ & $\begin{array}{l}\text { Enhancement according to } \\
\text { necrosis and inflammation: } \\
\text { Hypoenhancement in } \mathrm{CP}\end{array}$ \\
\hline Neuroendocrine tumors & $\begin{array}{l}\text { Hypoechoic } \\
\text { Well delineated } \\
\text { Undilated Wirsung duct } \\
\text { Rare vascular invasion } \\
\text { Metastases }\end{array}$ & $\begin{array}{l}\text { Rarely detectable } \\
\text { tumor vessels }\end{array}$ & Hyperenhancing tumors \\
\hline Cystadenomas & $\begin{array}{l}\text { Small cysts }(<3 \mathrm{~cm}) \\
\text { Small size calcifications } \\
\text { Fibrous septa } \\
\text { Undilated Wirsung duct }\end{array}$ & $\begin{array}{l}\text { Undetectable tumor } \\
\text { vessels }\end{array}$ & $\begin{array}{l}\text { Hyperenhancing tumors } \\
\text { Arteries accompanying the } \\
\text { fibrous septa }\end{array}$ \\
\hline Cystadenocarcinomas & $\begin{array}{l}\text { Large size cysts }(>5 \mathrm{~cm}) \\
\text { Echoic areas inside the cyst } \\
\text { and septaUndilated Wirsung } \\
\text { duct Metastases }\end{array}$ & $\begin{array}{l}\text { Rarely detectable } \\
\text { chaotic tumor } \\
\text { vessels }\end{array}$ & $\begin{array}{l}\text { Enhancing echoic areas inside the } \\
\text { cyst }\end{array}$ \\
\hline Pseudocysts & $\begin{array}{l}\text { Anechoic, well delineated lesion } \\
\text { Signs of acute/chronic pancreatitis } \\
\text { Signs of bleeding/calcifications } \\
\text { Accompanying changes of the } \\
\text { adjacent bowel }\end{array}$ & $\begin{array}{l}\text { Rarely detectable } \\
\text { tumor vessels in } \\
\text { young lesions }\end{array}$ & Unenhancing walls \\
\hline
\end{tabular}

Table 1. Differential diagnosis of pancreatic masses $[1,9,45,46]$.

Several studies proved the utility of CEUS for the characterization of pancreatic tumors $[2,9$, $17,47-49]$. The accuracy for the diagnosis of solid pancreatic lesions varies from $91.7 \%$ [2] to $92.9 \%$ [17] or $93.8 \%$ in other series [48]. 
In a recent meta-analysis that included 23 CEUS studies, the pooled estimate of CEUS sensitivity for the diagnosis of ductal adenocarcinoma was 0.89 (95\% CI, 0.85-0.92), while the average specificity was 0.84 (95\% CI, 0.77-0.89). For the differentiation of neoplastic and nonneoplastic lesions, the reported pooled sensitivity and specificity were 0.95 (95\% CI, $0.93-$ $0.96)$ and 0.72 (95\% CI, 0.58-0.83) [50].

\section{Contrast-enhanced endoscopic ultrasound (CE-EUS)}

CE-EUS combines the benefits of high-resolution US to those of a contrast-enhanced imaging method. It has two subtypes with similar performance: contrast-enhanced endoscopic Doppler ultrasound (which uses a high mechanical index for examination and no special software) and contrast-enhanced low mechanical index endoscopic ultrasound (in which the examination is made in contrast mode) [51]. CE-EUS in Doppler mode is useful to differentiate between ductal adenocarcinomas (in which only arterioles are seen] and pseudotumoral chronic pancreatitis (in which both arterioles and venules can be identified by CE-EUS Doppler) [52, 53].

CE-EUS with low mechanical index has been used similarly to transabdominal CEUS to differentiate between pseudotumoral $\mathrm{CP}$ and ductal adenocarcinoma, but also to guide FNA in order to avoid avascular areas, improving the diagnostic accuracy of FNA [54]. Quantitative postprocedural assessment of uptake of the contrast agent has been proven useful to improve the accuracy of CE-EUS with low mechanical index for differentiating between the two entities $[55,56]$.

As a conclusion, we must cite the EFSUMB guidelines on the nonhepatic use of CEUS, which state that CEUS is useful to improve characterization of ductal adenocarcinoma (A;1b); to differentiate between pseudocysts and cystic tumors (A;1b); to differentiate vascular (solid) from avascular (liquid/necrotic) components of a lesion (A;1b); to better define the dimensions and margins of a lesion, including its relationship with adjacent vessels $(B ; 2 b)$; to help the choice for a next imaging technique $(C ; 5)[1]$.

\section{Author details}

Roxana Șirli* and Alina Popescu

*Address all correspondence to: roxanasirli@gmail.com

Department of Gastroenterology and Hepatology, "Victor Babeș” University of Medicine and Pharmacy Timișoara, Romania 


\section{References}

[1] Piscaglia F, Nolsoe C, Dietrich CF, Cosgrove DO, Gilja OH, Bachmann Nielsen M, et al. The EFSUMB guidelines and recommendations on the clinical practice of contrast enhanced ultrasound (CEUS): update 2011 on non-hepatic applications. Ultraschall Med 2012; 33: 33-59. doi:10.1055/s-0031-1281676

[2] D'Onofrio M, Barbi E, Dietrich CF, Kitano M, Numata K, Sofuni A, et al. Pancreatic multicenter ultrasound study (PAMUS). Eur J Radiol 2012; 81: 630-638. doi:10.1016/ j.ejrad.2011.01.053

[3] Jakobsen JA, Oyen R, Thomsen HS, Morkos SK, Members of Contrast Media Safety Committee of European Society of Urogenital Radiology (ESUR). Safety of ultrasound contrast agents. Eur Radiol 2005; 15: 941-945. doi:10.1007/s00330-004-2601-0

[4] Piscaglia F, Bolondi L, Italian Society of Ultrasound in Medicine and Biology (SIUMB) Study Group on Ultrasound Contrast Agents. The safety of Sonovue in abdominal applications: retrospective analysis of 23188 investigations. Ultrasound Med Biol 2006; 32: 1369-1375. doi:10.1016/j.ultrasmedbio.2006.05.031

[5] Dillman JR, Ellis JH, Cohan RH, Strouse PJ, Jan SC. Frequency and severity of acute allergic-like reactions to gadolinium-containing i.v. contrast media in children and adults. AJR Am J Roentgenol 2007; 189: 1533-1538.

[6] Dean KE, Starikov A, Giambrone A, Hentel K, Min R, Loftus M. Adverse reactions to intravenous contrast media: an unexpected discrepancy between inpatient and outpatient cohorts. Clin Imaging Sep-Oct 2015; 39(5): 863-5. doi:10.1016/j.clinimag. 2015.04.014

[7] Caschera L, Lazzara A, Piergallini L, Ricci D, Tuscano B, Vanzulli A. Contrast agents in diagnostic imaging: present and future. Pharmacol Res Aug 2016; 110: 65-75. doi: 10.1016/j.phrs.2016.04.023

[8] Thorelius L. Usefulness of contrast-enhanced ultrasound in the characterization of pancreatic and renal masses. In: Albrecht T, Thorelius L, Solbiati L, Cova L, Frauscher F, ed. Contrast-enhanced Ultrasound in Clinical Practice. Springer, 2005, p. 25-35. DOI: 10.1007/88-470-0357-1_2

[9] Rickes S, Mönkemüller K, Malfertheiner P. Acute severe pancreatitis: contrast-enhanced sonography. Abdom Imaging 2007; 32: 362-364. doi:10.1007/s00261-007-9250-0

[10] Foitzik T, Bassi DG, Schmidt J, Lewandrowski KB, Fernandez-del Castillo C, Rattner $\mathrm{DW}$, et al. Intravenous contrast medium accentuates the severity of acute necrotizing pancreatitis in the rat. Gastroenterology 1994; 106: 207-214. doi:10.1016/S00165085(94)95457-7 
[11] Foitzik T, Bassi DG, Fernandez-del Castillo C, Warshaw AL, Rattner DW. Intravenous contrast medium impairs oxygenation of the pancreas in acute necrotizing pancreatitis in the rat. Arch Surg 1994; 129: 706-711. doi:10.1001/archsurg.1994.01420310038006

[12] Schmidt J, Hotz HJ, Foitzik T, Ryschich E, Buhr HJ, Warshaw A, et al. Intravenous contrast medium aggravates the impairment of pancreatic microcirculation in necrotizing pancreatitis in the rat. Ann Surg 1995; 221: 257-264. doi:10.1097/00000658199503000-00007

[13] Carmona-Sanchez R, Uscanga L, Bezaury-Rivas P, Robles-Díaz G, Suazo-Barahona J, Vargas-Vorácková F. Potential harmful effect of iodinated intravenous contrast medium on the clinical course of mild acute pancreatitis. Arch Surg 2000; 135: 1280-1284. doi: 10.1001/archsurg.135.11.1280

[14] Uhl W, Roggo A, Kirschstein T, Anghelacopoulos SE, Gloor B, Müller CA, et al. Influence of contrast-enhanced computed tomography on course and outcome in patients with acute pancreatitis. Pancreas 2002; 24: 191-197. doi:10.1097/00006676200203000-00011

[15] Rickes S, Uhle C, Kahl S, Kolfenbach S, Monkemuller K, Effenberger O, et al. Echo enhanced ultrasound: a new valid initial imaging approach for severe acute pancreatitis. Gut 2006; 55: 74-78. doi:10.1136/gut.2005.070276

[16] Ripolles T, Martinez MJ, Lopez E, Castello I, Delgado F. Contrast-enhanced ultrasound in the staging of acute pancreatitis. Eur Radiol 2010; 20: 2518-2523. doi:10.1007/s00330010-1824-5

[17] Ardelean M, Sirli R, Sporea I, Bota S, Martie A, Popescu A, et al. Contrast enhanced ultrasound in the pathology of the pancreas - a monocentric experience. Med Ultrason 2014; 16(4): 325-331. doi:10.11152/mu.201.3.2066.164.mars12

[18] van Gulik TM, Reeders JW, Bosma A, Moojen TM, Smits NJ, Allema JH, et al. Incidence and clinical findings of benign, inflammatory disease in patients resected for presumed pancreatic head cancer. Gastrointest Endosc 1997; 46: 417-423. doi:10.1016/S00165107(97)70034-8

[19] Koito K, Namieno T, Nagakawa T, Morita K. Inflammatory pancreatic masses: differentiation from ductal carcinomas with contrast-enhanced sonography using carbon dioxide microbubbles. AJR Am J Roentgenol 1997; 169: 1263-1267. doi:10.2214/ ajr.169.5.9353439

[20] D'Onofrio M, Zamboni G, Tognolini A, Malago R, Faccioli N, Frulloni L, et al. Massforming pancreatitis: value of contrast-enhanced ultrasonography. World J Gastroenterol 2006; 12: 4181-4184. doi:10.3748/wjg.v12.i26.4181

[21] D'Onofrio M, Malagò R, Martone E, et al. Pancreatic pathology. In: Quaia E, ed. Contrast Media in Ultrasonography. Berlin, Heidelberg: Springer-Verlag, 2005; 335-347. 
[22] Azemoto N, Kumagi T, Yokota T, Hirooka M, Kuroda T, Koizumi M, et al. Utility of contrast-enhanced transabdominal ultrasonography to diagnose early chronic pancreatitis. Biomed Res Int 2015; 2015: 393124. doi:10.1155/2015/393124

[23] D'Onofrio M, Martone E, Malagò R, Faccioli N, Zamboni G, Comai A, et al. Contrastenhanced ultrasonography of the pancreas. JOP. J Pancreas (Online) 2007; 8: 71-76.

[24] Kersting S, Konopke R, Kersting F, Volk A, Distler M, Bergert H, et al. Quantitative perfusion analysis of transabdominal contrast-enhanced ultrasonography of pancreatic masses and carcinomas. Gastroenterology 2009; 137: 1903-1911. doi:10.1053/j.gastro. 2009.08.049

[25] Numata K, Ozawa Y, Kobayashi N, Kubota T, Shimada H, Nozawa A, et al. Contrastenhanced sonography of pancreatic carcinoma: correlations with pathological findings. J Gastroenterol 2005; 40: 631-640. doi:10.1007/s00535-005-1598-8

[26] Kitano M, Kudo M, Maekawa K, Suetomi Y, Sakamoto H, Fukuta N, et al. Dynamic imaging of pancreatic diseases by contrast enhanced coded phase inversion harmonic ultrasonography. Gut 2004; 53: 854-859. doi:10.1136/gut.2003.029934

[27] Klöppel G. Pathology of the pancreas. In: Baert AL, Van Hoe DG, ed. Radiology of the Pancreas. 2nd ed. Berlin, Heidelberg: Springer-Verlag, 1999: 69-100.

[28] Faccioli N, D'Onofrio M, Malago R, Zamboni G, Falconi M, Capelli P, et al. Resectable pancreatic adenocarcinoma: depiction of tumoral margins at contrast-enhanced ultrasonography. Pancreas 2008; 37: 265-268. doi:10.1097/MPA.0b013e31816c908b

[29] Grossjohann HS, Rappeport ED, Jensen C, Svendsen LB, Hillingsø JG, Hansen CP, et al. Usefulness of contrastenhanced transabdominal ultrasound for tumor classification and tumor staging in the pancreatic head. Scand J Gastroenterol 2010; 45: 917-924. doi: $10.3109 / 00365521003702718$

[30] Procacci C, Carbognin G, Accordini S, Biasiutti C, Bicego E, Romano L, et al. Nonfunctioning endocrine tumors of the pancreas: possibilities of spiral CT characterization. Eur Radiol 2001; 11: 1175-1183. doi:10.1007/s003300000714

[31] D'Onofrio M, Mansueto G, Falconi M, Procacci C. Neuroendocrine pancreatic tumor: value of contrast enhanced ultrasonography. Abdom Imaging 2004; 29: 246-258. doi: 10.1007/s00261-003-0097-8

[32] Malago R, D'Onofrio M, Zamboni GA, Faccioli N, Falconi M, Boninsegna L, et al. Contrast-enhanced sonography of nonfunctioning pancreatic neuroendocrine tumors. Am J Roentgenol 2009; 192: 424-430. doi:10.2214/AJR.07.4043

[33] Hammond N, Miller FH, Sica GT, Gore RM. Imaging of cystic disease of the pancreas. Radiol Clin North Am 2002; 40: 1243-1262. doi:10.1016/S0033-8389(02)00054-4 
[34] Rickes S, Wermke W. Differentiation of cystic pancreatic neoplasms and pseudocysts by conventional and echo-enhanced ultrasound. J Gastroenterol Hepatol 2004; 19: 761766. doi:10.1111/j.1440-1746.2004.03406.x

[35] Lewandrowski K, Lee J, Southern J, Centeno B, Warshaw A. Cyst fluid analysis in the differential diagnosis of pancreatic cysts: a new approach to the preoperative assessment of pancreatic cystic lesions. AJR Am J Roentgenol Apr 1995; 164(4): 815-819. doi: 10.2214/ajr.164.4.7537015

[36] Le Borgne J, de Calan L, Partensky C, the French Surgical Association. Cystadenomas and cystadenocarcinomas of the pancreas: a multiinstitutional retrospective study of 398 cases. Ann Surg 1999; 230(2): 152. doi:10.1097/00000658-199908000-00004

[37] Fugazzola C, Procacci C, Bergamo Andreis IA, Iacono C, Portuese A, Dompieri P, et al. Cystic tumors of the pancreas: evaluation by ultrasonography and computed tomography. Gastrointest Radiol 1991; 16: 53-61. doi:10.1007/BF01887305

[38] Sperti C, Cappellazzo F, Pasquali C, Militello C, Catalini S, Bonadimani B, et al. Cystic neoplasms of the pancreas: problems in differential diagnosis. Am Surg 1993; 59: 740 745 .

[39] Demos TC, Posniak HV, Harmath C, Olson MC, Aranha G. Cystic lesions of the pancreas. AJR Am J Roentgenol 2002; 179: 1375-1388. doi:10.2214/ajr.179.6.1791375

[40] Scott J, Martin I, Redhead D, Hammond P, Garden OJ. Mucinous cystic neoplasm of the pancreas: imaging features and diagnostic difficulties. Clin Radiol 2000; 55: 187-192. doi:10.1053/crad.1999.0341

[41] D'Onofrio M, Caffarri S, Zamboni G, Falconi M, Mansueto G. Contrast-enhanced ultrasonography in the characterization of pancreatic mucinous cystadenoma. J Ultrasound Med 2004; 23: 1125-1129.

[42] D'Onofrio M, Megibow AJ, Faccioli N, Malagò R, Capelli P, Falconi M, et al. Comparison of contrast-enhanced sonography and MRI in displaying anatomic features of cystic pancreatic masses. Am J Roentgenol 2007; 189: 1435-1442. doi:10.2214/AJR.07.2032

[43] Itoh T, Hirooka Y, Itoh A, Hashimoto S, Kawashima H, Hara K, et al. Usefulness of contrast-enhanced transabdominal ultrasonography in the diagnosis of intraductal papillary mucinous tumors of the pancreas. Am J Gastroenterol 2005; 100: 144-152. doi: 10.1111/j.1572-0241.2005.40726.x

[44] D'Onofrio M, Gallotti A, Pozzi Mucelli R. Imaging techniques in pancreatic tumors. Expert Rev Med Devices 2010; 7: 257-273. doi:10.1586/erd.09.67

[45] Rickes S, Unkrodt K, Ocran K, Neye H, Lochs H, Wermke W. Evaluation of Doppler ultrasonography criteria for the differential diagnosis of pancreatic tumors. Ultraschall Med 2000; 20: 253-258. doi:10.1055/s-2000-9124

[46] Rickes S, Flath B, Unkrodt K, Ocran K, Neye H, Lochs H. Pancreatic metastases of renal cell carcinomas - evaluation of the contrast behaviour at echo-enhanced power Doppler 
sonography in comparison to primary pancreatic tumors. Z Gastroenterol 2001;39:571578. doi:10.1055/s-2001-16690

[47] D'Onofrio M, Gallotti A, Principe F, Mucelli RP. Contrastenhanced ultrasound of the pancreas. World J Radiol 2010; 2: 97-102. doi:10.4329/wjr.v2.i3.97

[48] Dietrich CF, Braden B, Hocke M, Ott M, Ignee A. Improved characterization of solitary solid pancreatic tumours using contrast enhanced transabdominal ultrasound. J Cancer Res Clin Oncol 2008; 134: 635-643. doi:10.1007/s00432-007-0326-6

[49] Ardelean M, Sirli R, Sporea I, Bota S, Danila M, Popescu A, et al. The value of contrastenhanced ultrasound in the characterization of vascular pattern of solid pancreatic lesions. Med Ultrason 2015; 17(1): 16-21. doi:10.11152/mu.2013.2066.171.mars

[50] D'Onofrio M, Biagioli E, Gerardi C, Canestrini S, Rulli E, Crosara S, et al. Diagnostic performance of contrast-enhanced ultrasound (CEUS) and contrast-enhanced endoscopic ultrasound (ECEUS) for the differentiation of pancreatic lesions: a systematic review and meta-analysis. Ultraschall Med 2014; 35(6): 515-21. doi:10.1055/s-00341385068

[51] Dietrich CF, Sharma M, Hocke M. Contrast-enhanced endoscopic ultrasound. Endosc Ultrasound 2012; 1(3): 130-6. doi:10.7178/eus.03.003

[52] Hocke M, Schulze E, Gottschalk P, Topalidis T, Dietrich CF. Contrast-enhanced endoscopic ultrasound in discrimination between focal pancreatitis and pancreatic cancer. World J Gastroenterol 2006; 12(2): 246-50. doi:10.3748/wjg.v12.i2.246

[53] Săftoiu A, Iordache SA, Gheonea DI, Popescu C, Malos A, Gorunescu F, et al. Combined contrast-enhanced power Doppler and real-time sonoelastography performed during EUS, used in the differential diagnosis of focal pancreatic masses (with videos). Gastrointest Endosc 2010; 72(4): 739-47. doi:10.1016/j.gie.2010.02.056

[54] Seicean A, Badea R, Moldovan-Pop A, Vultur S, Botan EC, Zaharie T, et al. Harmonic contrast-enhanced endoscopic ultrasonography for the guidance of fine-needle aspiration in solid pancreatic masses. Ultraschall Med 2015. [Epub ahead of print]. doi: 10.1055/s-0035-1553496

[55] Seicean A, Badea R, Stan-Iuga R, Mocan T, Gulei I, Pascu O. Quantitative contrastenhanced harmonic endoscopic ultrasonography for the discrimination of solid pancreatic masses. Ultraschall Med 2010; 31(6): 571-6. doi:10.1055/s-0029-1245833

[56] Săftoiu A, Vilmann P, Dietrich CF, Iglesias-Garcia J, Hocke M, Seicean A, et al. Quantitative contrast-enhanced harmonic EUS in differential diagnosis of focal pancreatic masses (with videos). Gastrointest Endosc 2015; 82(1): 59-69. doi:10.1016/j.gie. 2014.11.040 